\title{
microRNA expression profile in stage III colorectal cancer: Circulating miR-18a and miR-29a as promising biomarkers
}

\author{
ANNA BRUNET VEGA ${ }^{1}$, CARLES PERICAY $^{1,2}$, IRENE MOYA $^{2}$, ANNA FERRER $^{3,4}$, EMMA DOTOR $^{2}$, ALEYDIS PISA $^{2}$, \\ ÀLEX CASALOTS ${ }^{5}$, XAVIER SERRA-ARACIL ${ }^{6}$, JOAN-CARLES OLIVA $^{7}$, ANNA RUIZ $^{8}$ and EUGENI SAIGÍ ${ }^{2}$ \\ ${ }^{1}$ Department of Oncology Research, Parc Taulí Foundation - University Institute UAB, Corporació Sanitària Parc Taulí Hospital, \\ Parc Taulí s/n, 08208 Sabadell; ${ }^{2}$ Oncology Service, Corporació Sanitària Parc Taulí Hospital, Parc Taulí s/n, 08208 Sabadell; \\ ${ }^{3}$ Genomics Unit, Centre for Genomic Regulation (CRG), 08003 Barcelona; ${ }^{4}$ Universitat Pompeu Fabra (UPF), 08003 Barcelona; \\ ${ }^{5}$ Department of Pathology, Corporació Sanitària Parc Taulí Hospital, Parc Taulí s/n, 08208 Sabadell; ${ }^{6}$ Department of General \\ and Digestive Surgery, Corporació Sanitària Parc Taulí Hospital, Parc Taulí s/n, 08208 Sabadell; ${ }^{7}$ Statistical Unit, \\ Parc Taulí Foundation - University Institute UAB, Corporació Sanitària Parc Taulí Hospital, Parc Taulí s/n, \\ 08208 Sabadell; ${ }^{8}$ Genetic Laboratory, UDIAT-CD, Corporació Sanitària Parc Taulí Hospital, \\ Parc Taulí s/n, 08208 Sabadell, Barcelona, Spain
}

Received February 12, 2013; Accepted April 15, 2013

DOI: $10.3892 /$ or.2013.2475

\begin{abstract}
Biomarkers that can facilitate disease detection, staging and prediction of outcome are highly desirable to improve survival and to help determine optimized treatment for colorectal cancer patients. microRNAs (miRNAs) are small non-coding RNAs that play a crucial role in gene regulatory networks. The deregulation of miRNA expression has been found in several types of cancer and may represent a novel class of cancer biomarkers. Our aim was to determine the miRNA signature of stage III colorectal cancer (CRC) tumors and to identify potential circulating miRNAs that may represent noninvasive biomarkers in CRC patients. Genome-wide microarray analysis of miRNA expression was performed on 12 paired tumor and non-tumor formalin-fixed paraffin-embedded tissues from stage III CRC patients. A selection of differentially overexpressed miRNAs was validated by quantitative real-time polymerase chain reaction (qRT-PCR) and determined in the serum of a set of 56 individuals (30 stage III CRC patients and 26 healthy individuals). Using 1.5-fold expression difference as a cut-off level, 43 miRNAs were identified as differentially expressed in tumor versus normal tissue. Using reverse transcription and qRT-PCR, 11 miRNAs (miR-135b, miR-141, miR-18a, miR-20a, miR-21, miR-224, miR-29a, miR-31, miR-34a, miR-92a and miR-96) were confirmed as significantly overexpressed in tumor samples when compared with normal samples. We were able to detect 9 of these 11 miRNAs in serum samples from CRC patients
\end{abstract}

Correspondence to: Dr Anna Brunet Vega, Department of Oncology Research, Parc Taulí Foundation - University Institute UAB, Corporació Sanitària Parc Taulí Hospital, Parc Taulí s/n, 08208 Sabadell, Barcelona, Spain

E-mail: abrunetv@tauli.cat

Key words: microRNA, colorectal cancer, serum biomarker and healthy individuals. Serum levels of miR-18a and miR-29a were significantly higher in CRC patients when compared to levels in the controls $(\mathrm{p}<0.05)$. In conclusion, this study identified a substantial number of miRNAs which were differentially expressed in stage III colorectal tumors. Moreover, the findings provide relevant information concerning overexpressed tumoral miRNAs as potential circulating biomarkers and highlight serum miR-18a and miR-29a as promising biomarkers for the screening and monitoring of CRC patients.

\section{Introduction}

Colorectal cancer (CRC) is one of the most commonly diagnosed cancers and is a common cause of cancer-related deaths in developed countries (1). Prognosis strongly depends on tumor stage at presentation. The 5-year overall survival ranges from $93 \%$ for stage I patients to $8 \%$ for stage IV patients (2). Currently, the only curative treatment is surgical resection, with a modest survival benefit from chemotherapy, particularly in stage III disease. Stage III disease is defined by the presence of positive lymph nodes. The impact of adjuvant chemotherapy (CT) for patients with stage III CRC has been addressed in several clinical trials and practice-based studies demonstrating a benefit of adjuvant therapy in these patients $(3,4)$. However, current clinical experience with certain patients that may not be suitable for CT-based therapies (due to age, comorbid disease and/or potential treatment-related toxicity) faces a significant number of stage III CRC patients who do not suffer from relapse in the absence of adjuvant therapy. To date, substantial efforts have been given to the identification of clinicopathological and molecular parameters that improve the prognostic stratification of patients. To date, there are no available biomarkers to predict CRC recurrence.

microRNAs (miRNAs) are a newly discovered class of short 22 nucleotide non-coding RNAs that repress protein translation through binding to target mRNAs. Bioinformatics 
and cloning studies have estimated that miRNAs may regulate $30 \%$ of all human genes and control hundreds of gene targets (5). There is growing evidence that expression of miRNAs plays an important role in various cellular processes commonly implicated in cancer, such as differentiation, invasion, cell growth, angiogenesis and epithelial-mesenchymal transition (6-8). Data from several studies show that different miRNAs are aberrantly expressed in various hematological and solid malignant diseases including CRC (9-11). Functional studies indicate that miRNAs have both tumor-suppressive and oncogenic potential in human cancer (12) and can control various aspects of cancer biology such as chemoresistance (13) and metastasis (14). Accordingly, an increasing number of pro-metastatic and anti-metastatic miRNAs have been identified from expression profiling of tumor versus non-tumor tissue (15).

Accumulating data indicate that miRNAs are present in body fluids including blood plasma and serum, urine, saliva and semen $(16,17)$. Circulating miRNAs are stable and have been revealed as successful biomarkers for different malignancies including solid tumors $(16,18)$. Mammalian cells in culture have also been reported to export miRNAs into the extracellular environment $(19,20)$. To date, the discovery of miRNAs as novel biomarkers in serum or plasma have opened up a new and interesting field in the screening and monitoring of cancer patients.

In the present study, we aimed to establish comprehensive miRNA expression patterns in stage III CRC patients. Using microarray profiling we identified differentially expressed upregulated and downregulated miRNAs between CRC tissues and paired neighboring noncancerous colorectal tissues. To investigate candidate circulating cancer-associated miRNAs we determined the expression profile of upregulated tumoral miRNAs in serum samples of stage III CRC patients and healthy individuals.

\section{Patients and methods}

Ethics statement. The study was approved by the institutional medical ethics boards of our hospital (Corporació Sanitària Parc Taulí, Institut Universitari Parc Taulí-UAB). Serum samples of the patients and control were prospectively obtained, and both gave written informed consent to participate in the study. The tissue samples were obtained from formalin-fixed paraffin-embedded (FFPE) blocks stored in the Pathology Department of the hospital. For these patients, informed consent was not obtained in accordance with the Spanish Law 14/2007 for Biomedical Research. According to the law, archive samples may be used for biomedical research without informed consent when obtaining informed consent is not possible or extremely difficult, provided that i) the investigation performed is of general interest, ii) there is no previous declaration of the patient against the use of the samples for research, iii) data confidentiality is granted and iv) the ethics committee of the hospital evaluates and approves the study protocol.

Patients and samples. For the microarray analysis, 12 FFPE stage III colonic tumor tissue specimens and their surrounding non-cancerous normal mucosa were collected from 12 CRC patients who underwent surgical resection between 2000 and 2005 at the Department of Oncology at the hospital of Sabadell (Corporació Sanitària Parc Taulí, Institut Universitari Parc Taulí-UAB), Barcelona, Spain. Tumors were staged according to the Tumor-Node-Metastasis (TNM) staging system.

Peripheral blood samples ( $4 \mathrm{ml} /$ individual) were collected from 56 individuals: 30 stage III CRC patients and 26 healthy volunteers (13 female and 13 male with a median age of $64.1 \pm 7.4$ years) with no prior history of solid or hematological malignancy. No neoadjuvant therapy was used at the time when the blood samples were obtained.

Clinical and pathologic data were extracted from the medical records and reviewed for the purpose of this study. Detailed patient information is documented in Table I.

Sample processing and RNA isolation. miRNA-enriched total RNA from tissue specimens was extracted using RecoverAll ${ }^{\mathrm{TM}}$ Total Nucleic Acid isolation kit for FFPE samples (Ambion, Austin, TX, USA) following the manufacturer's protocol. Purity and concentration of the RNA were determined from OD260/280 readings using a spectrophotometer. RNA integrity was determined using the Bioanalyzer 2100 (Agilent Technologies Inc., Santa Clara, CA, USA).

A blood sample from each participant was collected in a serum clot activator tube (Vacuette ref. 454067) before surgical resection and centrifuged at $3,500 \mathrm{x}$ g for $10 \mathrm{~min}$ at $4^{\circ} \mathrm{C}$ to spin down the blood cells. Serum was then aliquoted and stored at $-80^{\circ} \mathrm{C}$ until use. RNA was isolated from $200 \mu \mathrm{l}$ serum using TRIzol LS (Invitrogen Life Technologies, Paisley, UK) as described by the manufacturer and finally eluted into a $50-\mu l$ volume of RNA-free water.

Microarray analysis. Microarray analysis was carried out by an external core facility (www.crg.es) using Agilent Human miRNA microarray including 887 human miRNAs, representing the miRBase set v14.0 (Agilent Technologies, Palo Alto, CA, USA).

Total RNA (100 ng) was labeled using Agilent miRNA Complete Labeling and Hyb kit (Agilent, cat. no. 5190-0456) following the manufacturer's instructions. Briefly, total RNA from each sample was dephosphorylated using calf intestinal alkaline phospatase (CIP), and cyanine 3-pCp was then ligated using the T4 RNA ligase. Labeled samples were dried and resuspended in $18 \mu \mathrm{l}$ of nuclease-free water and co-hybridized with in situ hybridization buffer for $20 \mathrm{~h}$ at $55^{\circ} \mathrm{C}$ on Agilent Human miRNA microarray release 14.0, 8x15K (G4471A029297). Arrays were washed at room temperature for $5 \mathrm{~min}$ in Gene Expression Wash Buffer 1 and $5 \mathrm{~min}$ at $37^{\circ} \mathrm{C}$ in Gene Expression Wash Buffer 2 (both from Agilent Technologies Inc.). Slides were scanned using Agilent's High-Resolution Scanner (G2539A) at $5 \mu \mathrm{m}$ resolution and quantified using Feature Extraction software (Agilent Technologies Inc.).

Quantification of miRNAs by $q R T-P C R$. Quantitative real-time polymerase chain reaction (qRT-PCR) was used to validate miRNAs upregulated in the microarray experiment data to determine the level of the same miRNAs in serum samples of CRC patients and healthy controls.

cDNA synthesis was performed using miRCURY LNA ${ }^{\mathrm{TM}}$ Universal RT microRNA PCR kit (Exiqon A/S, Vedbaek, 
Table I. Demographic and clinicopathological characteristics of the colorectal cancer patients.

\begin{tabular}{|c|c|c|}
\hline Variables & Tissue samples & Serum samples \\
\hline No. of patients & 12 & 30 \\
\hline Median age (years \pm SD) & $78.3 \pm 8$ & $68.1 \pm 11$ \\
\hline \multicolumn{3}{|l|}{ Gender } \\
\hline Male & 9 & 18 \\
\hline Female & 3 & 12 \\
\hline \multicolumn{3}{|l|}{ Tumour size (cm) } \\
\hline$<4$ & 2 & 12 \\
\hline$\geq 4$ & 10 & 18 \\
\hline \multicolumn{3}{|l|}{ Degree of differentiation } \\
\hline Poor & 7 & 23 \\
\hline Moderate-Well & 5 & 7 \\
\hline \multicolumn{3}{|l|}{ Vascular invasion } \\
\hline Yes & 4 & 10 \\
\hline No & 8 & 20 \\
\hline \multicolumn{3}{|l|}{ Perineural invasion } \\
\hline Yes & 4 & 14 \\
\hline No & 8 & 16 \\
\hline \multicolumn{3}{|l|}{ T stage } \\
\hline $\mathrm{T} 3$ & 5 & 20 \\
\hline $\mathrm{T} 4$ & 7 & 10 \\
\hline \multicolumn{3}{|l|}{$\mathrm{N}$ stage } \\
\hline N1 & 6 & 18 \\
\hline $\mathrm{N} 2$ & 6 & 12 \\
\hline \multicolumn{3}{|l|}{ M stage } \\
\hline M0 & 12 & 30 \\
\hline \multicolumn{3}{|l|}{ Clinical stage } \\
\hline III & 12 & 30 \\
\hline \multicolumn{3}{|l|}{ Localization } \\
\hline Left & 6 & 9 \\
\hline Right & 5 & 17 \\
\hline Rectum & 1 & 4 \\
\hline
\end{tabular}

Denmark) according to the manufacturer's instructions. Briefly, $20 \mathrm{ng}$ of total RNA from tissue or $4 \mu \mathrm{l}$ of total RNA from $200 \mu \mathrm{l}$ of serum was polyadenylated, and cDNA was synthesized using a poly( $\mathrm{T})$ primer with a $3^{\prime}$ degenerate anchor and a 5' universal tag. Then, cDNA served as a template for miRNA qPCR using FastStart Universal SYBR-Green Master (Rox) (Roche Applied Science, Indianapolis, IN, USA; cat no. 04913850001) and Exiqon-validated specific primer sets. The amplification profile was denatured in 96-well optical plates at $95^{\circ} \mathrm{C}$ for $10 \mathrm{~min}$, followed by 40 cycles of $95^{\circ} \mathrm{C}$ for $10 \mathrm{sec}$ and $60^{\circ} \mathrm{C}$ for $60 \mathrm{sec}$. At the end of the PCR cycles, melting curve analyses were performed. For each sample, both the cDNA synthesis and real-time PCR assays were performed in duplicate in a final volume of $20 \mu \mathrm{l}$ using an Applied Biosystems 7500 Sequence Detection system (Applied Biosystems, Foster City, CA, USA).
Data analysis and statistics. For statistical analysis of microarray data, extracted intensities were background corrected using the normexp method with an offset of 50 (21). To assure comparability across samples, we used quantile normalization on background corrected $\log 2$-transformed intensities. Microarray probes were collapsed to miRNAs by taking the median intensity of the respective probes per miRNA. For determining differentially regulated miRNAs, moderated t-tests were applied using limma (22). miRNAs with FDR adjusted $\mathrm{p}$-value $<5 \%$ and additionally a fold-change $(\mathrm{FC})>1.5$ in absolute value were selected as the relevant ones. All statistical analyses were performed with the Bioconductor project in the R statistical environment (23).

According to Exiqon's guidelines for microRNA qPCR in serum/plasma, we chose miR-16, miR-103 and miR-let-7a as reference miRNAs for normalization of expression levels. Using DataAssist ${ }^{\mathrm{TM}}$ Software v3.0 (Applied Biosystems) the fold-change in the miRNA expression was calculated by the equation $2^{-\Delta \Delta C T}$ (24). Paired t-test was performed and $\mathrm{p}$-values were adjusted using the Benjamini and Yekutieli false discovery rate method (25).

\section{Results}

Differentially expressed miRNAs in stage III CRC tumors and adjacent non-tumor tissues. Comparison of the expression profiles of 887 human miRNAs between normal and tumor tissue revealed a total of 43 differentially expressed miRNAs between tumors and corresponding non-tumor samples with a fold change $(\mathrm{FC})>1.5$ and adjusted $\mathrm{p}$-value below the threshold level of 0.05 . This miRNA signature was able to separate the tumors from the normal group into two main unsupervised hierarchial clusters shown in Fig. 1. The first cluster comprised all of the non-tumor samples and one tumor sample (case 3). The second cluster included 11 of the 12 tumor samples grouped together. Among these differentially expressed miRNAs, 30 miRNAs showed increased expression in colon tumors in relation to normal colon tissue, with miR-21 being the most significantly upregulated miRNA in the tumors at 3.6-fold. Expression of miR-20a, miR-19b, miR-17, miR-92a, miR-19a, miR-92a, mapping to the same region of chromosome 13, were highly correlated with a FC $>2$. Additionally, 13 miRNAs showed decreased expression in colon tumors, with miR-145 and miR-1 being the most downregulated at -5.2 -fold and -3.1 -fold, respectively.

miRNA selection and $q R T-P C R$ validation. Thirteen overexpressed miRNAs in CRC tumors were selected for validation using qRT-PCR in the same cohort of 12 paired samples investigated initially by the microarray assay. Ten highly upregulated miRNAs (FC >1.9, p<0.05): miR-135b, miR-142-3p, miR-20a, miR-21, miR-223, miR-224, miR-29a, miR-34a, miR-92a, miR-96, along with 3 miRNAs which were selected on the basis of previous studies supporting their involvement in CRC prognosis: miR-141 (FC 1.76, p=0.04), miR-18a (FC $1.36, \mathrm{p}=0.0035)$ and miR-31 (FC 1.57, p=0.0035). Table II summarizes the 13 selected miRNAs and their experimentally observed associated target genes. Notably, the determined heat map using only unsupervised clustering of these 13 miRNAs showed that the entire normal tissue profile clustered together 
Table II. List of 13 deregulated miRNAs identified by array profiling in stage III CRC and selected for experimental validation by qRT-PCR.

\begin{tabular}{|c|c|c|c|}
\hline miRNA & $\begin{array}{l}\text { Chromosome } \\
\text { localization }\end{array}$ & $\mathrm{FC}$ & Experimentally observed target genes \\
\hline hsa-miR-21 & $17 q 23$ & 3.56 & $\begin{array}{l}\text { ACTA2, APAF1, BMPR2, BTG2, CDC25A, CDK6, CDKN1A, E2F1, } \\
\text { FAS, FASLG, IL6R, JAG1, MARCKS, NFIB, PIK3R1, PTEN, } \\
\text { SERPINB5, SOCS5, SPRY2, TGFBR2, TIMP3, TPM1 }\end{array}$ \\
\hline hsa-miR-135b & $1 \mathrm{q} 32.1$ & 2.76 & APC, JAK2, RUNX2, SMAD5 \\
\hline hsa-miR-20a & $13 \mathrm{q} 31.3$ & 2.48 & $\begin{array}{l}\text { BAMBI, BCL2, BCL2L11, BMPR2, CCND1, CDKN1A, E2F1, } \\
\text { ESR1, IL8, JAK1, MICA, NCOA3, PPARG, PTEN, RB1, RBL2, } \\
\text { RUNX1, S1PR1, STAT3, TGFBR2, TP63, VEGFA, VIM }\end{array}$ \\
\hline hsa-miR-223 & $\mathrm{Xq} 22$ & 2.40 & IRS1, MEF2C, RHOB, STMN1, VIM \\
\hline hsa-miR-224 & $\mathrm{Xq} 28$ & 2.18 & AP2M1, KLK1 \\
\hline hsa-miR-92a & $\begin{array}{l}13 q 31.3 \\
\mathrm{Xq} 26.2\end{array}$ & 2.08 & BCL2L11, BMPR2, CDKN1A, CDKN1C, ITGA5, MAP2K4 \\
\hline hsa-miR-96 & $\begin{array}{l}1 \mathrm{q} 32.2 \\
7 \mathrm{q} 32.2\end{array}$ & 2.05 & FOXO1, IRS1, MITF, RYK \\
\hline hsa-miR-142-3p & $17 q 22$ & 2.01 & BCL2L1, LIFR \\
\hline hsa-miR-34a & $1 \mathrm{p} 36.22$ & 1.92 & $\begin{array}{l}\text { AXIN2, BCL2, CCND1, CD47, CDK6, CREB1, DLL1, E2F3, HDAC1, JAG1, } \\
\text { MAP2K1, MET, MYC, MYCN, NOTCH1, NOTCH2, VEGFA, WNT1 }\end{array}$ \\
\hline hsa-miR-29a & $7 q 32.3$ & 1.90 & $\begin{array}{l}\text { ACVR2A, CAV2, CDC42, CDK6, COL1A1, COL1A2, COL3A1, DNMT3A, } \\
\text { DNMT3B, DUSP2, HDAC4, MCL1, PIK3R1, PPM1D, TCL1A, TGFB3, TPM1, YY1 }\end{array}$ \\
\hline hsa-miR-141 & $12 \mathrm{p} 13.31$ & 1.76 & BAP1, CTNNB1, CYP1B1, EPHA2, EXOC5, MAP2K4, PRKACB, TGFB2 \\
\hline hsa-miR-31 & $9 \mathrm{p} 21.3$ & 1.57 & HIF1A, ITGA5, PDGFB, PPP2R2A, RDX, RHOA \\
\hline hsa-miR-18a & $13 q 31.3$ & 1.36 & BCL2L11, CDKN1A, CTGF, E2F1, ESR1, THBS1, TPG3 \\
\hline
\end{tabular}

miRNA, microRNA; CRC, colorectal cancer; qRT-PCR, quantitative real-time polymerase chain reaction; FC, fold change. Observed predicted targets of differentially expressed miRNAs were assessed using Ingenuity Pathway Analysis (IPA; Ingenuity Systems, Redwood City, CA, USA).

in one group (including sample 3) and separately from the group of colon tumor samples (data not shown).

Statistically significant increases in 11 miRNAs (miR135b, miR-141, miR-18a, miR-20a, miR-21, miR-224, miR-29a, miR-31, miR-34a, miR-92a, miR-96) were validated by qRT-PCR in the 12 CRC tissue samples compared with non-cancerous adjacent colorectal mucosa. No significant differences were observed for miR-142-3p and miR-223 (data not shown).

Evaluation of miRNAs in serum samples. Employing qRT-PCR assay we then compared the expression levels of the 11 validated miRNAs in serum samples from 56 individuals: 30 stage III CRC patients and 26 healthy donors. Our data reflected that the relative levels of miR-135b, miR-141 and miR-224 were not consistently measurable in serum. miR-31 showed two peaks in the derivative melting curve and was therefore not considered for the analysis. Eventually, 7 miRNAs (miR-18a, miR-20a, miR-21, miR-29a, miR-34a, miR-92a and miR-96) were efficiently extracted and amplified from the serum of stage III CRC patients and healthy individuals. The levels of expression of these miRNAs are shown in Table III. We found that the expression levels of miR-18a and miR-29a, normalized to the level of miR-103, miR-16 and miR-let-7a endogenous controls, were significantly elevated (Benjamini-Hochberg adjusted $\mathrm{p}<0.05$ ) in the serum of CRC patients compared with the healthy individuals (Fig. 2).

\section{Discussion}

The identification of molecular markers that can predict prognosis is an important aim in cancer research. Accumulating evidence suggess that miRNAs play an active role in controlling the development, differentiation and cancer progression. miRNAs are well preserved in tissue samples even after formalin-fixation and paraffin-embedding and can be efficiently extracted and evaluated in tumors and also in body fluids such as serum or plasma. Therefore, currently, there is a growing interest in the study of miRNAs as molecular biomarkers with potential clinical applicability to tumor classification, cancer diagnosis and disease follow-up.

In the present study, we used a commercial platform including 887 validated human miRNAs to investigate miRNA expression profiling in stage III colon adenocarcinomas 


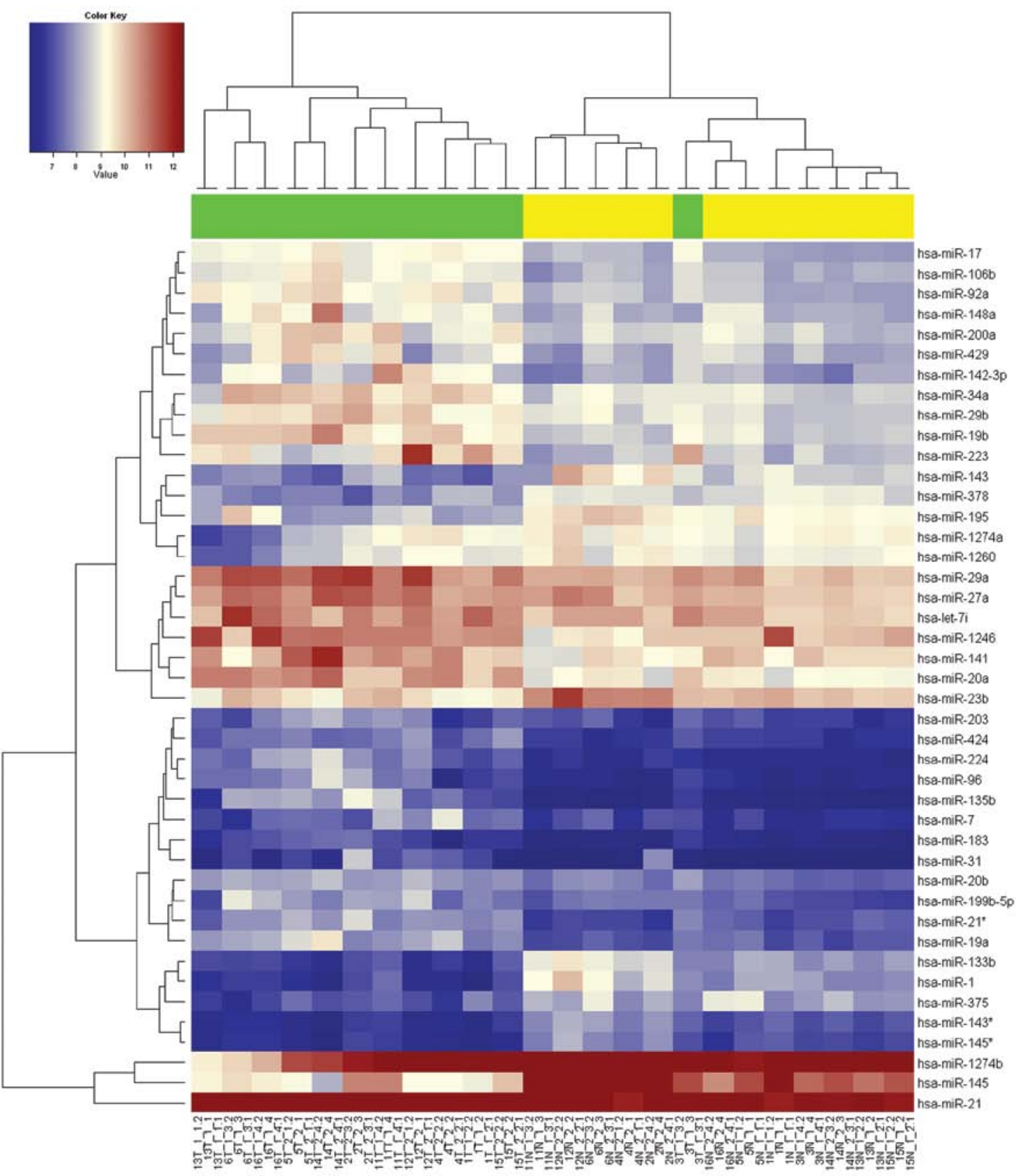

Figure 1. Unsupervised hierarchial clustering of 12 paired stage III colorectal tumor and non-tumor tissues. Using a 1.5-fold expression difference (Padj $<0.05$ ) as a cut-off level, 43 miRNAs were found to be differentially expressed in tumor vs. normal tissue. The relative miRNA expression changes are expressed by a color gradient intensity scale, as shown at the top. The lightest blue color indicates a maximal decrease and the lightest red color indicates a maximal increase in gene expression. Each column represents a separate CRC sample and each row a single miRNA. Samples are grouped into two main clusters: green cluster represents tumor tissue samples $(\mathrm{T})$ and the yellow cluster represents normal colorectal tissue $(\mathrm{N})$.

miR-29a

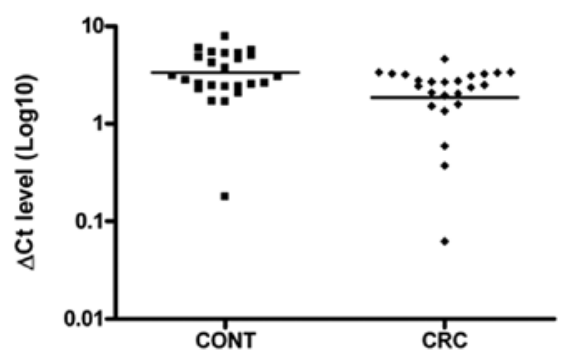

miR-18a

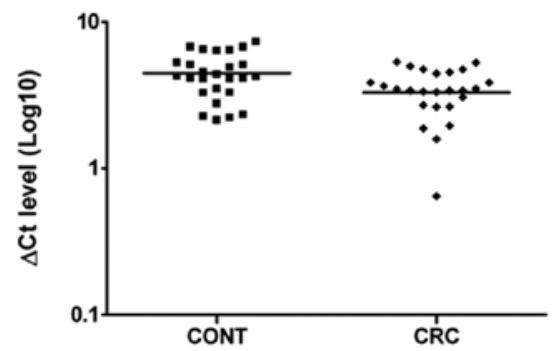

Figure 2. Differential expression of miR-18a and miR-29a in serum samples of colorectal cancer patients (CRC) and healthy individuals (CONT). The normalized $\mathrm{Ct}$ values (show as $\Delta \mathrm{Ct}$ values with respect to reference miR-103, miR-16 and miR-let-7a) indicate higher expression levels in CRC cases when compared with the controls. The horizontal line represents the mean value. 
Table III. Mean fold change of the 7 miRNAs identified in the serum samples of CRC patients and healthy individuals.

\begin{tabular}{llcc}
\hline miRNA & \multicolumn{1}{c}{ Type } & Mean FC & P-value \\
\hline miR-18a & Target & 2.229 & 0.038 \\
miR-20a & Target & 1.378 & 0.593 \\
miR-21 & Target & 2.081 & 0.087 \\
miR-29a & Target & 2.811 & 0.038 \\
miR-34a & Target & 3.205 & 0.055 \\
miR-92a & Target & 0.444 & 0.087 \\
miR-96 & Target & 2.267 & 0.107 \\
miR-103 & Selected control & 1.324 & 0.496 \\
miR-16 & Selected control & 0.829 & 0.593 \\
miR-let-7a & Selected control & 0.910 & 0.702 \\
\hline
\end{tabular}

CRC, colorectal cancer; FC, fold-change. p-values were adjusted using the Benjamini-Hochberg false discovery rate.

and adjacent non-tumor tissues. In agreement with previous reports, our data confirmed that numerous miRNAs are aberrantly expressed in CRC in relation to normal tissue. Heat map and cluster analyses showed that miRNA signatures were able to separate cancer tissue and non-tumor tissue in most cases. We found concordant expression of previously reported miRNAs altered in CRC (i.e. downregulation of miR-143, miR.145 or miR-195 and upregulation of miR-21, miR-17-92 cluster or miR-135b). Moreover, we identified several other miRNAs which have not previously been reported in CRC, probably since they have been more recently included in array platforms (i.e. downregulation of miR-1274a, miR-1274b, miR-1260 and upregulation of miR-1246, miR-199b-5p, miR-29b). The current results support other published studies and contribute to the better characterization of differentially expressed miRNAs in stage III CRC tumors.

The discovery of the presence of miRNAs in blood (serum or plasma) has generated great scientific and clinical interest. The expression pattern of miRNAs in the circulation could be relevant to identify the high risk of disease progression in CRC. While many studies have shown the overexpression or downregulation of specific miRNAs in CRC tissue or cell lines, there is less data concerning circulating tumor-associated miRNAs.

Therefore, we investigated the circulating levels of 11 miRNAs overexpressed in tumors and identified in serum samples of stage III CRC patients and healthy individuals. Our results identified at least 2 highly overexpressed miRNAs (miR-135b and miR-224) in tumor tissue that have not been previously detected in the serum of patients nor in healthy individuals. Future studies determining the cellular localization of these 2 miRNAs as well as an increasing understanding of miRNA transportation outside cells are warranted to address the absence of circulating miR-135b and miR-224.

The present study identified 7 miRNAs that were stable and detectable in all serum samples analyzed. To our knowledge, this is the first study to investigate circulating levels of miR-18a, miR-142-3p, miR-20a and miR-223 in serum samples of CRC patients. Our results identified miR-18a and miR-29a, whose expression was found to differ significantly between CRC patients and normal donor sera. miR-18a, located at region $13 \mathrm{q} 31.3$, is one of the 6 miRNAs encoded by the miR-17-92 cluster. This cluster, also named oncomir-1, is known as a body of oncogenic miRNAs and typically exhibits increased expression in tumors, including CRC. Previous studies have reported significantly elevated levels of both miR-17-3p and miR-92 in the blood of patients with CRC $(26,27)$. In our study, no significant difference was observed in the levels of miR-92a between CRC patients and healthy individuals $(\mathrm{RQ}=1.7362, \mathrm{p}=0.453)$. In contrast, we identified miR-18a as another miRNA included in the same cluster which was significantly elevated in the serum of patients with $C R C(R Q=2.670, p<0.031)$. Of interest, in CRC, miR-18a* targets specifically on K-Ras (28), and has recently been found to be significantly higher in the plasma of pancreatic cancer patients than that in the controls (29). To our knowledge, our study is the first report to demonstrate miR-18a as a potential novel non-invasive biomarker for CRC patients.

Consistent with another published report, we found that serum miR-29a was significantly higher in CRC cancer patients than in healthy individuals (27). Results from recent studies revealed miR-29a as a significant potential candidate prognostic biomarker in CRC patients. Higher expression of miR-29a has been reported in stage II CRC tumoral samples, and was associated with a longer disease-free survival (30). Furthermore, upregulation of miR-29a was observed in colorectal tumor tissue from liver metastatic (CRLM) patients when compared with non-metastatic CRC patients. Other authors, demonstrated that serum miR-29a can discriminate CRLM patients from non-metastatic CRC patients with a specificity and sensitivity higher than serum CEA $(60 \%$ sensitivity and 34\% specificity) (31). In addition, one study that combined in silico analysis and an empirical experiment also indicated miR-29a as a potential biomarker to predict the early recurrence of CRC. All of these findings together indicate that the circulating level of miR-29a warrants further testing as a promising serum biomarker of prognosis in CRC patients.

In summary, the data presented in this study confirm that miRNAs are differentially expressed between colonic tumor tissues and adjacent tissues. Our results provide a list of 43 miRNAs that are differentially expressed between stage III CRC tissue and corresponding normal tissue, which could contribute to further explain the role of miRNAs in colonic tumors with lymph node metastasis. Our data showed that not all overexpressed tumor miRNAs are detectable in serum fluids. Further investigation of miR-18a and miR-29a, preferably in large prospective studies, to validate their potential use as circulating biomarkers in CRC patients is warranted.

\section{Acknowledgements}

We are grateful to all the patients and healthy individuals who kindly allowed samples to be collected and used in this study. The authors would also like to acknowledge the nursing staff Francisca Cuadras-Lacasa and Marta Fernández-Ros of Corporació Sanitària Parc Taulí, Institut Universitari Parc Taulí-UAB for their continued technical assistance and collaboration. Finally, we thank Sarah Bonnin of the CRG Genomics Unit for the excellent technical assistance. This 
study was supported by 'Fundació Benèfica Privada Barnola Vallribera Sant Josep' from Sabadell and by 'Fundació Parc Taulí - Institut Universitari UAB' of the Sabadell Hospital (CIR2010029).

\section{References}

1. Weitz J, Koch M, Debus J, Höhler T, Galle PR and Büchler MW: Colorectal cancer. Lancet 365: 153-165, 2005.

2. O'Connell JB, Maggard MA and Ko CY: Colon cancer survival rates with the new American Joint Committee on Cancer 6th edition staging. J Natl Cancer Inst 96: 1420-1425, 2004.

3. No authors listed: Efficacy of adjuvant fluorouracil and folinic acid in B2 colon cancer. International Multicentre Pooled Analysis of B2 Colon Cancer Trials (IMPACT B2) Investigators. J Clin Oncol 17: 1356-1363, 1999.

4. No authors listed: Efficacy of adjuvant fluorouracil and folinic acid in colon cancer. International Multicentre Pooled Analysis of Colon Cancer Trials (IMPACT) investigators. Lancet 345: 939-944, 1995.

5. Wu H, Neilson JR, Kumar P, et al: miRNA profiling of naïve, effector and memory CD8 T cells. PLoS One 2: e1020, 2007.

6 . Wiemer EA: The role of microRNAs in cancer: no small matter. Eur J Cancer 43: 1529-1544, 2007.

7. Calin GA and Croce CM: MicroRNA signatures in human cancers. Nat Rev Cancer 6: 857-866, 2006.

8. Esquela-Kerscher A and Slack FJ: Oncomirs - microRNAs with a role in cancer. Nat Rev Cancer 6: 259-269, 2006.

9. Michael MZ, O' Connor SM, van Holst Pellekaan NG, Young GP and James RJ: Reduced accumulation of specific microRNAs in colorectal neoplasia. Mol Cancer Res 1: 882-891, 2003.

10. Cummins JM, He Y, Leary RJ, et al: The colorectal microRNAome. Proc Natl Acad Sci USA 103: 3687-3692, 2006.

11. Bandrés E, Cubedo E, Agirre X, et al: Identification by real-time PCR of 13 mature microRNAs differentially expressed in colorectal cancer and non-tumoral tissues. Mol Cancer 5: 29, 2006.

12. Garzon R, Calin GA and Croce CM: MicroRNAs in cancer. Annu Rev Med 60: 167-179, 2009.

13. Ma J, Dong C and Ji C: MicroRNA and drug resistance. Cancer Gene Ther 17: 523-531, 2010.

14. Hurst DR, Edmonds MD and Welch DR: Metastamir: the field of metastasis-regulatory microRNA is spreading. Cancer Res 69: 7495-7498, 2009.

15. Zhang H, Li Y and Lai M: The microRNA network and tumor metastasis. Oncogene 29: 937-948, 2010.

16. Mitchell PS, Parkin RK, Kroh EM, et al: Circulating microRNAs as stable blood-based markers for cancer detection. Proc Natl Acad Sci USA 105: 10513-10518, 2008.
17. Weber JA, Baxter DH, Zhang S, et al: The microRNA spectrum in 12 body fluids. Clin Chem 56: 1733-1741, 2010.

18. Chen X, Ba Y, Ma L, et al: Characterization of microRNAs in serum: a novel class of biomarkers for diagnosis of cancer and other diseases. Cell Res 18: 997-1006, 2008.

19. Valadi H, Ekström K, Bossios A, Sjöstrand M, Lee JJ and Lötvall JO: Exosome-mediated transfer of mRNAs and microRNAs is a novel mechanism of genetic exchange between cells. Nat Cell Biol 9: 654-659, 2007.

20. Kosaka N, Iguchi H, Yoshioka Y, Takeshita F, Matsuki Y and Ochiya T: Secretory mechanisms and intercellular transfer of microRNAs in living cells. J Biol Chem 285: 17442-17452, 2010.

21. Ritchie ME, Silver J, Oshlack A, et al: A comparison of background correction methods for two-colour microarrays. Bioinformatics 23: 2700-2707, 2007.

22. Smyth GK: Linear models and empirical bayes methods for assessing differential expression in microarray experiments. Stat Appl Genet Mol Biol 3: Article 3, 2004.

23. Gentleman RC, Carey VJ, Bates DM, et al: Bioconductor: open software development for computational biology and bioinformatics. Genome Biol 5: R80, 2004.

24. Schmittgen TD and Livak KJ: Analyzing real-time PCR data by the comparative C(T) method. Nat Protoc 3: 1101-1108, 2008

25. Benjamini Y, Drai D, Elmer G, Kafkafi N and Golani I: Controlling the false discovery rate in behavior genetics research. Behav Brain Res 125: 279-284, 2001.

26. Ng EK, Chong WW, Jin H, et al: Differential expression of microRNAs in plasma of patients with colorectal cancer: a potential marker for colorectal cancer screening. Gut 58: 1375-1381, 2009.

27. Huang Z, Huang D, Ni S, Peng Z, Sheng W and Du X: Plasma microRNAs are promising novel biomarkers for early detection of colorectal cancer. Int J Cancer 127: 118-126, 2010.

28. Tsang WP and Kwok TT: The miR-18a* microRNA functions as a potential tumor suppressor by targeting on K-Ras. Carcinogenesis 30: 953-959, 2009.

29. Morimura R, Komatsu S, Ichikawa D, et al: Novel diagnostic value of circulating miR-18a in plasma of patients with pancreatic cancer. Br J Cancer 105: 1733-1740, 2011.

30. Weissmann-Brenner A, Kushnir M, Lithwick Yanai G, et al: Tumor microRNA-29a expression and the risk of recurrence in stage II colon cancer. Int J Oncol 40: 2097-2103, 2012.

31. Wang LG and Gu J: Serum microRNA-29a is a promising novel marker for early detection of colorectal liver metastasis. Cancer Epidemiol 36: e61-e67, 2012. 